\title{
Settlement and bearing capacity of the circular foundation
}

\author{
Zaven Ter-Martirosyan ${ }^{1}$, and Vitalii Sidorov ${ }^{1, *}$ \\ ${ }^{1}$ Moscow State University of Civil Engineering, Yaroslavskoe shosse, 26, Moscow, 129337, Russia
}

\begin{abstract}
In process of designing foundations of circular form in plane, as well as bored piles of circular cross section, there is important to quantify the stress-strain state and the bearing capacity of the soils interacted with the foundations, as well as under the toe of piles. The article presents the formulation and solution of the problem of determining the initial critical load at the base of the circular foundation, which is also relevant for the design case of the soil under the toe of a circular bored pile. It is shown that the condition of limiting equilibrium arises both under the center of the loaded area and on the contour of the circular loading area, and primarily on the contour. A comparative estimate showed that the ratio of the initial critical load to the base soil of the circular foundation under the center of the site is 2 times greater than the corresponding load for the point at the edge of the site.
\end{abstract}

\section{Introduction}

The problem of the bearing capacity of soils under the foundation was considered earlier by many authors [1], but without taking into account the internal friction of the soil. There is also a three-term empirical formula [2] for determining the load-bearing capacity of base soils, taking into account the angle of internal friction and adhesion through dimensionless coefficients $\mathrm{N}_{\gamma}, \mathrm{N}_{\mathrm{q}}, \mathrm{N}_{\mathrm{c}}$. For plane strain problem, there is a solution of L. Prandtl and $\mathrm{G}$. Reisner, taking into account the depth of the load, the angle of friction of the soil and its cohesion [1]. To determine the initial critical and critical loads within the framework of the plane problem of Flaman, there is a solution of N.P. Puzyrevskii [3]. In this paper, we consider the formulation and solution of the problem of determining the initial critical load at the base and under the edge of the circular foundation, taking into account the depth $d$ of its laying, as well as the angle of internal friction and cohesion, based on the solution of the problem of stress-strain state in the soil under the influence of a uniformly distributed constant load by the area of a circle of diameter $2 \mathrm{a}$.

\footnotetext{
* Corresponding author: vitsid@mail.ru
} 


\section{Initial equations}

It is known that under the influence of a uniformly distributed load intensity $p=$ const of the area of a circle of diameter $2 \mathrm{a}$, a complex stress-strain state arises [4-10]. As a result, a system of stresses, deformations and displacements, including vertical stresses $\sigma_{\mathrm{z}}$, radial $\sigma_{\mathrm{r}}$ and tangential $\sigma_{\mathrm{t}}$, arises and we have the following stresses on the $\mathrm{z}$ axis $(\mathrm{z}=0)$ :

$$
\begin{gathered}
\sigma_{\mathrm{z}}=p\left[1-z^{3}\left(a^{2}+z^{2}\right)^{-3 / 2}\right] \\
\sigma_{r}=\sigma_{t}=p\left[1+2 v-2(1+v)\left(a^{2}+z^{2}\right)^{-1 / 2} \cdot z+\left(a^{2}+z^{2}\right)^{-3 / 2} \cdot z^{3}\right]
\end{gathered}
$$

The expression for the draft in the center of the square takes the form:

$$
\mathrm{S}_{0}=\frac{(1-v) \cdot p \cdot a}{G}=\frac{2\left(1-v^{2}\right) p \cdot a}{E},
$$

where $v, G, E$ - Poisson's coefficient, shear modulus and soil modulus of deformation, respectively.

On the radius $0<\mathrm{r}<\mathrm{a}$ and if $\mathrm{z}=0$, we have the boundary conditions:

$$
\begin{gathered}
\sigma_{z}=p, \sigma_{r}=\sigma_{t}=\frac{(1+2 v) p}{2} \\
S_{a}=\frac{2}{\pi} S_{0},
\end{gathered}
$$

where $S_{a}$ - the value of the settlement at a point at a distance $a$ from the center of the loaded area.

On the radius $0<\mathrm{r}<\infty$ and if $\mathrm{z}=0$, we have another boundary conditions:

$$
\sigma_{z}=0, \sigma_{r}=-\sigma_{t}=\frac{(1-2 v) p \cdot \mathrm{a}^{2}}{2 \cdot r^{2}}
$$
arises:

It follows from these expressions that on the $\mathrm{z}$ axis at $\mathrm{z}=0$ the maximum shear stress

$$
\tau_{\max }=\left(\sigma_{z}-\sigma_{t}\right) / 2
$$

or

$$
\tau_{\max }=\frac{p}{4}\left[-1+2 v+2(1+v) \cdot z\left(a^{2}+z^{2}\right)^{-1 / 2}+3 z^{3}\left(a^{2}+z^{2}\right)^{-3 / 2}\right]
$$

And besides $\tau_{\max }$ reaches its maximum value at depth $\mathrm{z}_{\max }$ :

$$
z_{\max }=a \sqrt{\frac{2(1+v)}{7-2 v}}
$$

At $v=0,3, \mathrm{Z}_{\max }=0,637 a$, i.e. $\tau / \tau_{\max }=0,33 p$. 
It can be assumed that the limiting state arises at a depth $z_{\max }(9)$. However, taking into account the abrupt change in the radial and tangential stresses on the contour of the loaded area (6), when $\sigma_{t}$ becomes tensile, one should expect that the limiting state first of all will arise at the point with $\mathrm{z}=0$ and $\mathrm{r}=\mathrm{a}$. And it will penetrate into the base perpendicular to the plane $\mathrm{z}=0$. Obviously, it becomes necessary to determine the initial critical load from the conditions when the limiting state occurs under the center of the loaded area at $z=z_{\max }$ (9) and at the point $\mathrm{r}=\mathrm{a}, \mathrm{z}=0$. form:

To determine the limiting state, we use the Mohr-Coulomb strength condition in the

$$
\sin \varphi=\frac{\sigma_{1}-\sigma_{3}}{\sigma_{1}+\sigma_{3}+2 c \cdot \operatorname{ctg} \varphi}
$$

where $\sigma_{1}$ and $\sigma_{3}$ - maximum and minimum stress values under conditions of a triaxial stress state. In the case we are considering, this is $\sigma_{z}$ and $\sigma_{t}$ also for a point in the center of the area at a depth $\mathrm{z}=\mathrm{z}_{\max }$ and $\sigma_{\mathrm{r}}$ and $\sigma_{\mathrm{t}}$ for a point at $\mathrm{r}=\mathrm{a}$ at a depth $\mathrm{z}=0$.

\section{Statement and solution of the problem}

Suppose that the foundation of the circular section rests on the base soils at a depth $d$ from the surface of the soil half-space (Figure 1). At the base level of the foundation, the distributed load is affected by intensity $\mathrm{p}=$ const.

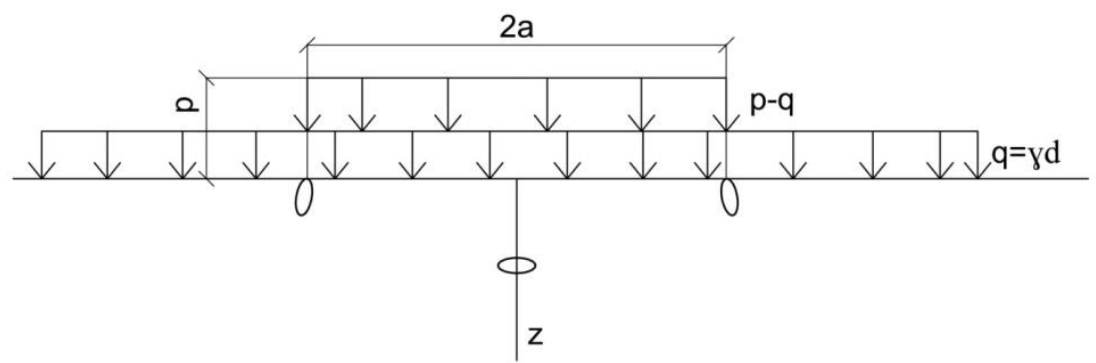

Fig. 1. Calculation scheme for determining the initial critical load under the center and under the edge of the circular foundation

First of all, consider the condition of the beginning of limiting equilibrium under the center of the loaded area. From formula (1-2), taking into account that $p^{\prime}=p-q$, we obtain for the depth $\mathrm{z}=\mathrm{z}_{\max }(9)$ :

$$
\begin{aligned}
& \sigma_{z}-\sigma_{t}=(p-q)\left[2(1+v)\left(a^{2}+z_{\max }^{2}\right)^{1 / 2} \cdot z_{\max }-2 z_{\max }^{3}\left(a^{2}+z_{\max }^{2}\right)^{-3 / 2}-2 v\right]= \\
& =(p-q) \cdot A \\
& \sigma_{z}+\sigma_{t}=(p-q)\left[2+2 v-2(1+v) z_{\text {max }}\left(a^{2}+z_{\max }^{2}\right)^{-1 / 2}\right]+2 q+2 \gamma \cdot z_{\max }, \\
& =(p-q) B-2 q
\end{aligned}
$$

where $A$ and $B$ - are constants, determined by the expressions in square brackets.

Substituting these values into condition (10), we obtain

$$
\sigma_{z}-\sigma_{t}=\left(\sigma_{z}+\sigma_{t}\right) \sin \varphi+2 c \cdot \cos \varphi
$$


Taking into account (11) and (12), the expression can be represented as:

$$
(p-q) A=\left[(p-q) B+2 q \cdot z_{\max }\right] \cdot \sin \varphi+2 c \cdot \cos \varphi
$$

It follows that the initial critical load at a depth $z=z_{\max }(9)$ is determined by an expression of the form:

$$
p_{H}=\frac{q(A-B \sin \varphi+2 \sin \varphi)+2 \gamma \cdot z_{\max } \cdot \sin \varphi+2 c \cdot \cos \varphi}{A-B \cdot \sin \varphi}
$$

Let us consider the condition for the appearance of limiting equilibrium on the contour of the loaded area, i.e. at the point $r=a, z=0$ on the basis of (6). Then we get:

$$
\begin{gathered}
\sigma_{z}-\sigma_{t}=(1-2 v)(p-q) \\
\sigma_{z}+\sigma_{t}=2 \cdot \gamma \cdot d
\end{gathered}
$$

Substituting these values into (10), we obtain

$$
(1-2 v)(p-q)=2 \gamma \cdot d \cdot \sin \varphi+2 c \cdot \cos \varphi
$$

It follows that the initial critical load $p_{\text {ic }}$ from the condition of the limiting state at the point $r=a, z=0$ is determined by an expression of the form:

$$
p_{\text {ic }}=\gamma_{d}+\frac{2 \gamma \cdot d \cdot \sin \varphi+2 c \cdot \cos \varphi}{1-2 v}
$$

Consider an example of calculating the initial critical load for a tape and circular foundation with the following initial data:

$\gamma=18 \kappa \mathrm{H} / \mathrm{m}^{3}, c=20 \kappa \mathrm{Pa}, \varphi=15^{\circ}, d=5 \mathrm{~m}$.

For a point on the edge of the foundation in accordance with Puzyrevsky's solution for a plane problem:

$$
p_{i c}=\frac{\pi \cdot(\gamma \cdot d+c \cdot \operatorname{ctg} \varphi)}{\operatorname{ctg} \varphi+\varphi-\pi / 2}+\gamma \cdot d=\frac{3.14 \cdot(18 \cdot 5+20 \cdot 3.73)}{3.73+0,262-1.57}+18 \cdot 5=303,6(\mathrm{kPa})
$$

By the formula (19) obtained:

$$
p_{н \kappa}=\gamma \cdot d+\frac{2 \gamma d \cdot \sin \varphi+2 c \cdot \cos \varphi}{1-2 v}=18 \cdot 5+\frac{2 \cdot 18 \cdot 5 \cdot \sin 15+2 \cdot 20 \cdot \cos 15}{1-2 \cdot 0.3}=303(\mathrm{kPa})
$$

Thus, the initial critical load for the circular foundation and for the strip foundation practically coincided.

We also calculate the initial critical load for the circular foundation for the point in the center of the loaded area by formula (15):

$$
p_{H}=\frac{18(0.49-1.19 \sin 15+2 \sin 15)+2 \cdot 18 \cdot 0.64 \cdot \sin 15+2 \cdot 20 \cdot \cos 15}{0.49-1.19 \cdot \sin 15}=597.7(\mathrm{kPa})
$$

The initial critical load in the center of the loaded circular area is 1.97 times larger than the corresponding value for the base boundary point. 
The conclusion that under the circular foundation the first points of plastic deformations appear more quickly and on the whole the zones of limiting equilibrium develop faster is also confirmed by the results of numerical simulation in the PLAXIS 3D presented below.
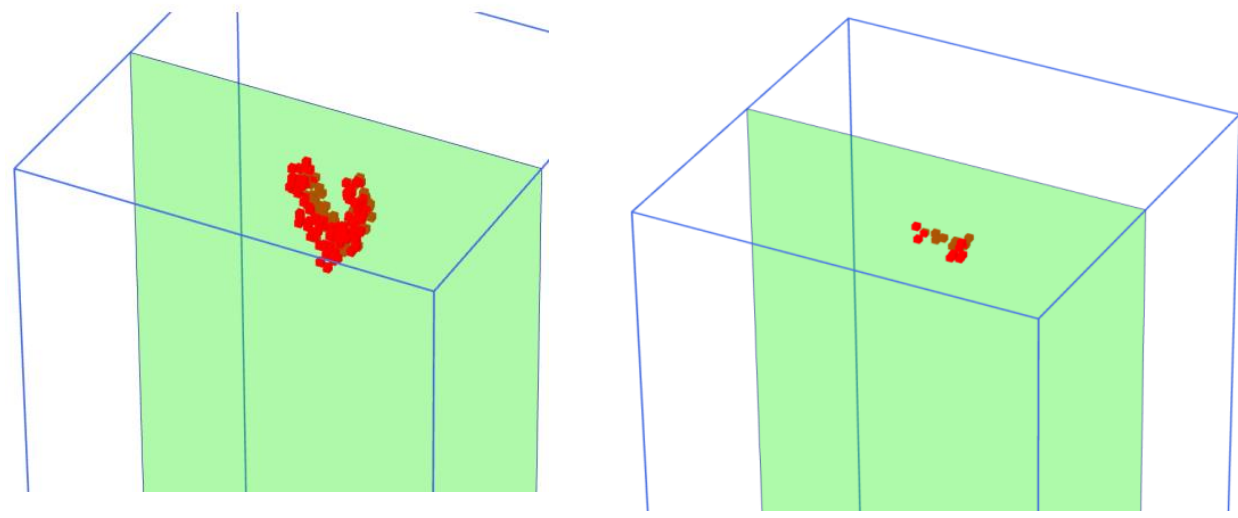

Fig. 2. The forms of the limit equilibrium zones in the base for the strip foundation (left) and the circular foundation (right) of the same width $\mathrm{d}=2 \mathrm{~m}$ for the same load $\mathrm{p}=300 \mathrm{kPa}$

\section{Conclusions}

1. The ratio of the initial critical load to the base soil of the circular foundation under the center of the site is 2 times greater than the corresponding load for the point on the edge of the site.

2. The formula for calculating the initial critical load for a circular foundation is given. A comparative estimate of the values shows that for a circular base this value is equal to the value for the case of a plane problem (the circular foundation), so the traditional PrandtlReisner solution in the case of a circular foundation is used correctly.

3. Numerical comparative modeling of the arising of zones of limit equilibrium at the base of the strip and circular foundations confirmed the assumption that zones under the strip foundation grows faster than under the circular one.

All tests were carried out using research equipment of The Head Regional Shared Research Facilities of the Moscow State University of Civil Engineering (RFMEFI59317X0006).

\section{References}

1. S.B. Ukhov, V.V. Semenov, V.V. Znamensky, Z.G. Ter-Martirosyan, S.N. Chernyshev, Soil mechanics, bases and foundations (Moscow, Association of construction higher education institutions, 175, 1994)

2. J.B. Hansen, Bulletin 28, Danish Geotechnical Institute, 5-11, (1970)

3. Z.G. Ter-Martirosyan, Mechanics of soil (Moscow, Association of construction higher education institutions, 550, 2009)

4. A.V. Pilyagin, Bulletin of the PNRPU. Constraction and architecture, 2, 158-162 (2014) 
5. Yu.F. Sukhodoev, Modern Technology, Materials and Design in Construction, 9, 2, 6570 (2010)

6. V.A. Mironov, O.E. Sofin, Bulletin of the TSTU, 8, 24-27 (2006)

7. V.M. Strulev, V.Yu. Voevodkin, Bulletin of the TSTU, 13, 3, 809-813 (2007)

8. M. Alla, Modern Technology, Materials and Design in Construction, 6, 1, 90-91, (2009)

9. A.Yu. Marshalka, I.G. Tkachev, M.A. Sharov, Actual science, 3, 3, 96-97 (2017)

10. R.A. Timchenko, A.V. Bogatynsky, Bulletin of the KRNU, 33, 4, 65-68 (2013)

11. R.F. Ganiev, S.R. Ganiev, V.P. Kasilov, A.P. Pustovgar, Wave Technology in Mechanical Engineering: Industrial Applications of Wave and Oscillation Phenomena, (Wiley, 2015)

12. K.S. Fahmi, M.Y. Fattah, A. Pustovgar, MATEC Web of Conferences, 170, 03001 (2018) 